Ann. Zootech., I977, 26 (4), 575-584.

\title{
Le transit digestif chez le Lapin. VIII. - Influence de la source de cellulose
}

\author{
F. LEBAS (r) (*), et J. P. LAPLACE $(* *)$
}

avec la collaboration technique de C. GFRMAIN

(*) Labovatoire de Recherches sur l'Élevage du lapin

(**) Laboratoive de Physiologie de la Nutrition

Centre national de Recherches zootechniques, I.N.R.A., 78350 Jouy on Josas (France)

\section{Résumé}

L'influence respective de trois régimes isocellulosiques fournis ad libitum, contenant soit de la paille d'orge, soit de la sciure de chêne, soit de la cellulose de bois purifiée, à l'égard du transit: digestif a été étudiée chez 24 lapins de race californienne, des deux sexes. Après une semaine d'habituation, ils ont reçu à l'âge de 8 semaines une dose de I 4 I Ce dont l'élimination par voie fécale a été suivie durant 72 heures.

Les résultats montrent que le transit est le plus rapide avec l'aliment contenant de la paille et le plus lent avec celui qui renferme de la cellulose de bois purifiée. Ce classement des 3 régimes fondé sur la rétention de I4ICe est systématiquement retrouvé pour l'ensemble des critères mesurés. Par conséquent le dosage de la cellulose brute ne rend pas compte des constituants membranaires éventuellement responsables de ces particularités. Diverses hypothèses explicatives sont proposées, en relation notamment avec la teneur en amidon et la concentıation énergétique des régimes.

\section{Introduction}

Une expérimentation antérieure (LEBAS, LAPIACE, I977) a montré que le transit digestif d'un radioélément est plus lent lorsque l'aliment est pauvre en cellulose, et que pour une teneur analogue en cellulose, la nature de celle-ci n'est pas indifférente. Cor,IN et al. (I976) ont aussi montré une amélioration des performances de croissance et une réduction des diarrhées lorsque l'aliment contient une proportion croissante de cellulose. Ces observations nous ont incités à préciser l'importance éventuelle d'une influence de la nature des fibres sur le transit digestif de lapins recevant des aliments isocellulosiques.

(I) Adresse actuelle : Centre de Rechetches de Toulouse, B. P. 12, 31320 Castanet-Tolosan. 


\section{Matériel et méthodes}

Au total 24 lapins, de race californienne, des 2 sexes ont été répartis en 3 lots de 8 animaux. A l'âge de 7 semaines, ils ont été placés en cage individuelle et ont reçu à volonté l'un des trois régimes expérimentaux définis au tableau I. La composition de ces derniers a été ajustée de façon à ce qu'ils soient isocellulosiques

TABLEAU I

Composition centésimale et composition analytique des aliments expérimentaux (en p. cent de l'aliment)

Centesimal and analytical composition of the experimental diets (per cent of feed)

\begin{tabular}{|c|c|c|c|}
\hline $\begin{array}{c}\text { Régime } \\
\text { Diet }\end{array}$ & $\begin{array}{l}\text { Paille } \\
\text { Straw }\end{array}$ & $\begin{array}{l}\text { Sciure de bois } \\
\text { Wood sawdust }\end{array}$ & $\begin{array}{c}\text { Cellulose de bois } \\
\text { Wood cellulose }\end{array}$ \\
\hline 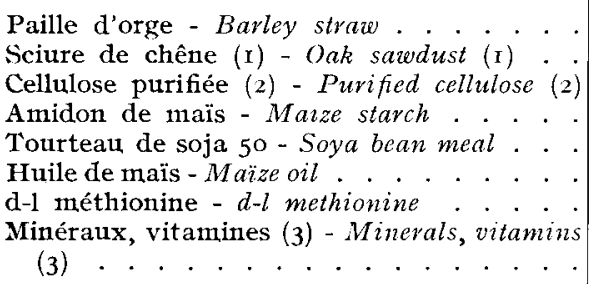 & $\begin{array}{r}33,0 \\
- \\
29,9 \\
32,0 \\
2,0 \\
0,1 \\
3,0\end{array}$ & $\begin{array}{r}-- \\
29,0 \\
33,9 \\
32,0 \\
2,0 \\
0,1 \\
3,0\end{array}$ & $\begin{array}{r}- \\
\overline{1}-\overline{5} \\
44,4 \\
32,0 \\
2,0 \\
0,1 \\
3,0\end{array}$ \\
\hline 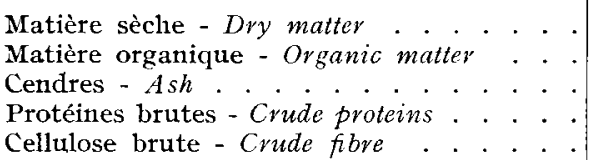 & $\begin{array}{r}85,1 \\
79,3 \\
5,8 \\
14,3 \\
19,3\end{array}$ & $\begin{array}{r}84,3 \\
79,7 \\
4,6 \\
14,7 \\
20,8\end{array}$ & $\begin{array}{r}86,5 \\
82,2 \\
4,3 \\
14,4 \\
20,2\end{array}$ \\
\hline
\end{tabular}

(I) Brute de sciage fin; particules de taille inféricure à $\mathbf{I}, 5 \mathrm{~mm}$. Produced by fine saring; particles smaller than $x, 5 \mathrm{~mm}$.

(2) "Colmacel $F_{2}$ ", Société Novacel, Paris.

(3) Colin, ARKhurst et LEBAs, I973.

(cellulose brute) et isoazotés. La cellulose de bois purifiée est produite à partir d'un mélange de bois blanes et de bois résineux. La sciure de chêne provient du travail de planches de chêne non traité. La paille d'orge d'hiver a été broyée au broyeur à marteaux (grille de $2 \mathrm{~mm}$ ) avant son incorporation dans 1'aliment. La recherche des tanins par la méthode officielle A.O.A.C. (I) a été effectuée sur ces trois sources de fibres. L,es teneurs enregistrées sont respectivement de $0,0-1,7-0,8$ pour la cellulose purifiée, la sciure de chêne, et la paille d'orge.

(I) A.O.A.C. = Association of Official Agricultural Chemists, Washington DC, C.S.A. 
A partir de la $8^{e}$ semaine d'âge, les fèces dures sont collectées selon le procédé antérieurement décrit (LEBAS, LAPLACE, I974) durant les 3 nycthémères consécutifs à l'administration à $9 \mathrm{~h} 30$ (I) d'une solution de I4I Ce (LAPLACE, LEBAS, RIOPEREZ, I974). Les fèces recueillies sont, après dessication, soumises au comptage de la radioactivité; au terme des 72 heures, les viscères digestifs font également l'objet d'un comptage par compartiment; le détail de la méthodologie mise en cuvre a été décrit antérieurement (LAPLACE, LEBAS, I975).

Les 24 lapins dont les résultats sont exposés dans le présent travail ont été retenus parmi 43 animaux mis en accoutumance à l'âge de 7 semaines. Les 9 lapins éliminés 1'ont été soit pour défaut d'adaptation au régime alimentaire $(n=\mathrm{I} 3)$ avec ingestion anormalement faible, soit en raison de la consistance trop molle des fèces produites $(n=6)$ interdisant une récolte horaire quatıtitative par le dispositif habituel. Ces contraintes impliquent que nous n'avons pu obtenir une parfaite homogénéité des poids vifs initiaux, ni une répartition équilibrée des deux sexes au sein de chaque groupe. Néanmoins, les poids vifs initiaux des lapins ne sont pas significativement différents les uns des autres.

\section{Résultats}

\section{I. - Croissances et consommations d'aliment}

Malgré une divergence temporaire du poids vif à la $8^{\mathbf{e}}$ semaine des groupes sciure/cellulose de bois, les poids vifs à 1'abattage ne diffèrent pas significativement. Globalement (tab1. 2) la vitesse de croissance moyenne des animaux des 3 groupes n'est pas statistiquement différente en raison de sa forte variabilité.

La consommation d'aliment (tabl. 3) en période diurne n'est pas sensiblement différente quel que soit l'aliment. Par contre, l'ingestion moyenne horaire du régime cellulose de bois est significativement plus faible en période nocturne que celle du régime à base de paille au cours des mêmes heures. Cette différence se répercute de façon significative sur la consommation moyenne quotidienne totale.

\section{2. - Excrétion des fèces dures}

La quantité totale de matière sèche excrétée sous forme de fèces dures (tabl. 4) est significativement plus faible pour les lapins ayant ingéré le régime cellulose de bois que pour leurs congénères des deux autres groupes. Cependant la quantité collectée par heure est identique pour les trois groupes $(3 \mathrm{~g} / \mathrm{h})$.

L'ingestion de l'aliment contenant de la paille conduit à l'émission de fèces significativement $(\mathrm{P}<\mathrm{O}, \mathrm{OOI})$ plus riches en eau.

Le nombre d'heures au cours desquelles est constatée une excrétion de fèces dures (tab1. 5) fait apparaître une réduction significative d'environ 4 heures de la durée de la période de production de crottes dures chez les lapins consommant l'aliment cellulose de bois. Mais cet abrégement de la période d'excrétion résulte surtout d'un morcellement plus que d'une contraction dans le temps (fig. I). La localisation de cette période dans le cycle de 24 heures n'est pas modifiée de manière importante. En effet les heures moyennes pondérées d'excrétion ne sont pas significativement différentes les unes des autres.

(r) 9 h 30 heure légale soit, lors de la réalisation de l'expérience, 8 h 30 luetre solaire. 
TABLEAU 2

Poids vits moyens et gain moyen quotidien de poids vif des lapins: moyenne et écart-type de la moyenne

Mean live weight and daily weight gain of the rabbits: mean and standard deviation of the mean

\begin{tabular}{|c|c|c|c|c|c|}
\hline \multicolumn{3}{|c|}{$\begin{array}{l}\text { Régime } \\
\text { Diet }\end{array}$} & $\begin{array}{l}\text { Paille } \\
\text { Straw }\end{array}$ & $\begin{array}{l}\text { Sciure de bois } \\
\text { Wood sawdust }\end{array}$ & $\begin{array}{l}\text { Cellulose de bois } \\
\text { Wood cellulose }\end{array}$ \\
\hline \multirow{3}{*}{$\begin{array}{c}\text { Poids vif à : } \\
\text { Live weight } \\
\text { at: } \\
\text { (g) }\end{array}$} & $\begin{array}{l}7^{\mathrm{e}} \text { semaine. } \\
7^{\text {th }} \text { week } .\end{array}$ & $\begin{array}{l}\cdot \cdot \cdot \\
\cdot \cdot \cdot\end{array}$ & $\begin{array}{r}3 \text { I } 5 \\
\text { I } 4\end{array}$ & $\begin{array}{r}265 \\
40 \\
\end{array}$ & $\begin{array}{l}\text { I } 34^{8} \\
\quad \text { I } 6\end{array}$ \\
\hline & $\begin{array}{l}8^{\mathrm{e}} \text { semaine. } \\
8^{\text {th }} \text { week . }\end{array}$ & $\begin{array}{l}\cdot \cdot \cdot \cdot \\
\cdot \cdot \cdot\end{array}$ & $\begin{array}{r}\text { I } 488 \\
39\end{array}$ & $\begin{array}{r}368 \\
58\end{array}$ & $\begin{array}{r}533 \\
27\end{array}$ \\
\hline & $\begin{array}{l}\text { Abattage } . \\
\text { Slaughter } .\end{array}$ & $\begin{array}{l}\cdot \cdot \cdot \cdot \\
\cdot \cdot \cdot\end{array}$ & $\begin{array}{r}\text { I } 634 \\
33\end{array}$ & $\begin{array}{r}532 \\
62\end{array}$ & $\begin{array}{r}645 \\
29\end{array}$ \\
\hline \multicolumn{3}{|c|}{$\begin{array}{l}\text { Gain de poids quotidien }(\mathrm{I}) \\
\text { Daily weight gain }(\mathrm{g}) \cdot . \\
.\end{array}$} & $\begin{array}{r}35,7 \\
4,8\end{array}$ & $\begin{array}{r}26,7 \\
2,5\end{array}$ & $\begin{array}{r}29,7 \\
2,7\end{array}$ \\
\hline
\end{tabular}

(r) De la $7^{e}$ semaine à l'abattage (ro jours). From the 7 th week to slaughter (ro days).

\section{TABLEAU 3}

Ingestion moyenne hovaive d'aliment $(g / h)$ au cours des périodes "diurne " et "nocturne " et quantité totale $(\mathrm{g})$ d'aliment ingéré au cours du cycle de $24 \mathrm{~h}:$ moyenne et écart-type de la moyenne - calculs effectués sur la base des 3 nycthémèves expérimentaux.

Mean hourly feed intake $(g / h)$ during "light" and "dark" periods and total daily feed intake during the whole $24 \mathrm{~h}$-cycle: mean and standard deviation of the mean, on the basis of the 3 experimental days.

\begin{tabular}{|c|c|c|c|c|c|c|c|c|c|c|c|}
\hline & $\begin{array}{l}\text { Période } \\
\text { Period }\end{array}$ & & & & & & & & 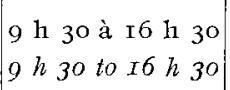 & 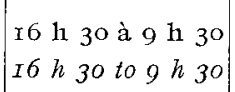 & 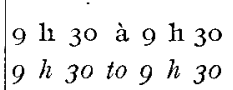 \\
\hline $\begin{array}{l}\text { Paille . . . . . } \\
\text { Straw . . . . }\end{array}$ & $\begin{array}{l}\cdot \cdot \cdot \cdot \\
\cdot \cdot \cdot\end{array}$ & . & $\dot{\cdot}$ & $\cdot$ & . & $\dot{r}$ & . $\cdot$ & $\dot{.}$ & $\begin{array}{l}3,90 \\
0,26\end{array}$ & $\begin{array}{l}6,39 a \\
0,59\end{array}$ & $\begin{array}{l}\text { I } 36,0 a \\
\text { I I, } 8\end{array}$ \\
\hline $\begin{array}{l}\text { Sciure de bois } \\
\text { Wood sawdust }\end{array}$ & $\begin{array}{lll}\cdot & \cdot & \cdot \\
. & \cdot & \cdot\end{array}$ & $\cdot$ & . & $\cdot$ & & . & · $\cdot$ &. & $\begin{array}{l}3,4 \text { I } \\
0,34\end{array}$ & $\begin{array}{l}5,85 a b \\
0,24\end{array}$ & $\begin{array}{l}\mathrm{I} 23,5 a b \\
\quad 6,0\end{array}$ \\
\hline $\begin{array}{l}\text { Cellulose de bois } \\
\text { Wood cellulose }\end{array}$ & $\begin{array}{lll}\cdot & \cdot & \cdot \\
\cdot & \cdot & \cdot\end{array}$ & . & . & $\dot{.}$ & & ${ }^{*}$ & .. & . & $\begin{array}{l}3,39 \\
0,44\end{array}$ & $\begin{array}{l}4,9 I \quad b \\
0,29\end{array}$ & $\begin{array}{c}107, \mathrm{I} b \\
8,9\end{array}$ \\
\hline $\begin{array}{l}\text { Eiffet régime. } \\
\text { Diet effect. . . }\end{array}$ & $\begin{array}{llll}\cdot & \cdot & \cdot & \cdot \\
. & \cdot & \cdot & .\end{array}$ & $\cdot$ & . & . & & $\cdot$ & . & & N.S. & $\mathrm{P}<0,05$ & $\mathrm{P}<0, \mathrm{IO}$ \\
\hline
\end{tabular}




\section{TABLEAU 4}

Quantités moyennes $(g)$ de matière sèche excrétécs sous forme de fècos dures au cours de chaque nycthémève expérimental, et au cours de la période totale (rapportée à $24 \mathrm{~h})$; tencur moyenne en matière sèche des fèces récoltées : moyenne et écart-type de la moyenne.

Mean quantities ( $g$ ) of dry matter excreted as hard faeces during each experimental day and duving the whole period (quantity per $24 \mathrm{~h}$ ); mean dry matter content of the collected facces: mean and standard deviation of the mean.

\begin{tabular}{|c|c|c|c|c|c|c|c|c|c|c|c|c|}
\hline & & $\begin{array}{l}\text { gim } \\
\text { giet }\end{array}$ & & & & & & & & $\begin{array}{l}\text { Paille } \\
\text { Straw }\end{array}$ & $\begin{array}{l}\text { Sciure de bois } \\
\text { Wood sawdust }\end{array}$ & $\begin{array}{l}\text { Cellulose de bois } \\
\text { H'ood cellulose }\end{array}$ \\
\hline $\begin{array}{l}\text { I }^{\mathrm{r}} \text { jour. . . } \\
I^{\text {st }} \text { day . . }\end{array}$ & $\cdot \cdot$ & $\begin{array}{l}\cdot . \\
\cdot \dot{ }\end{array}$ & . & . & $\dot{v}$ & & . & . : & . & $\begin{array}{c}39,5 a \\
3,0\end{array}$ & $\begin{array}{r}37,3 a \\
3,3\end{array}$ & $\begin{array}{c}24,1 \quad b \\
3,0\end{array}$ \\
\hline $\begin{array}{l}2^{\mathrm{e}} \text { jour } . \\
2 n d \text { day. } .\end{array}$ & $\begin{array}{l}\cdot \cdot \\
\cdot \cdot\end{array}$ & $\begin{array}{l}\cdot . \\
\cdot .\end{array}$ & . & . & $\dot{.}$ & . & . & . $\cdot$ & . & $\begin{array}{c}4 \mathrm{I}, 6 a \\
3,2\end{array}$ & $\begin{array}{r}38,6 a \\
3,8\end{array}$ & $\begin{array}{r}21,3 b \\
2,0\end{array}$ \\
\hline $\begin{array}{l}3^{\mathrm{e}} \text { jour } . \\
3^{\text {rd }} \text { day } . \\
.\end{array}$ & $\cdot \dot{ } \cdot$ & $\begin{array}{l}\cdot . \\
\cdot .\end{array}$ & . & . & . & $\cdot$ & $\cdot$ & . $\cdot$ & . & $\begin{array}{r}4 \mathrm{r}, 8 \quad a \\
2,5\end{array}$ & $\begin{array}{r}40,4 a \\
3,3\end{array}$ & $\begin{array}{rl}22,8 & b \\
1,8 & \end{array}$ \\
\hline $\begin{array}{l}\text { Période totale } \\
\text { Whole period }\end{array}$ & $\begin{array}{l}\cdot \cdot \cdot \\
\cdot \cdot\end{array}$ & & . & . & . & & & $\cdot \cdot$ & . & $\begin{array}{c}4 \mathrm{I}, 0 \quad a \\
\mathrm{I}, 7\end{array}$ & $\begin{array}{c}38,8 a \\
2,0\end{array}$ & $\begin{array}{r}22,7 b \\
\mathbf{1}, 3\end{array}$ \\
\hline $\begin{array}{l}\text { Teneur en mati } \\
\text { Dry matter con }\end{array}$ & $\begin{array}{l}\text { ère st } \\
\text { tent }\end{array}$ & che & • & - & . & . & . & . $\cdot$ & . & $\begin{array}{c}53,0 a \\
\mathrm{I}, 5\end{array}$ & $\begin{array}{c}61,3 b \\
1,2\end{array}$ & $\begin{array}{rl}6 \mathbf{1}, 7 & b \\
\mathbf{1}, 8 & \end{array}$ \\
\hline
\end{tabular}

\section{TABIEAU 5}

Nombre d'heures du nycthémève durant lesquelles une collecte de fèces dures a été réalisie, et heure moyenne d'excrétion fécale (en heures décimalisées) pondérée en fonction de la quantité de matière sèche émise: moyenne avec écart-type de la moyenne.

Number of hours with effective hard faeces excretion during the 24 period, and avorage hour of hard facces excretion (decimal hours): mean and standard deviation of the mean.

\begin{tabular}{|c|c|c|c|c|c|c|c|}
\hline \multicolumn{5}{|c|}{$\begin{array}{c}\text { Régime } \\
\text { Diet }\end{array}$} & $\begin{array}{l}\text { Paille } \\
\text { Straw }\end{array}$ & $\begin{array}{l}\text { Sciure de bois } \\
\text { Wood sawdust }\end{array}$ & $\begin{array}{l}\text { Cellulose de bois } \\
\text { Wood cellulose }\end{array}$ \\
\hline \multirow{3}{*}{$\begin{array}{l}\text { Nombre } \\
\text { d'heures } \\
\text { d'excrétion } \\
\text { Numbers } \\
\text { of hours } \\
\text { with fecal } \\
\text { excretion }\end{array}$} & $\begin{array}{l}\text { Jour I } \\
\text { Day I . }\end{array}$ & $\cdot$. & 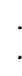 & $\dot{v}$ & $\begin{array}{c}13,6 a \\
0,4\end{array}$ & $\begin{array}{r}\mathrm{I} 3,0 a \\
0,5\end{array}$ & $\begin{array}{l}9,6 \quad b \\
\mathrm{I}, \mathrm{I}\end{array}$ \\
\hline & $\begin{array}{l}\text { Jour } 2 \\
\text { Day } 2\end{array}$ & . $\cdot$ & . & ${ }^{*}$ & $\begin{array}{c}\text { I } 4,0 a \\
0,8\end{array}$ & $\begin{array}{c}12,8 a \\
0,6\end{array}$ & $\begin{array}{ll}7,1 & b \\
0,7 & \end{array}$ \\
\hline & $\begin{array}{l}\text { Jour } 3 \\
\text { Day } 3\end{array}$ & .. & . & $\dot{v}$ & $\begin{array}{c}\text { I } 3,8 a \\
0,7\end{array}$ & $\begin{array}{rl}\mathbf{1} 2,8 & a \\
0,8 & \end{array}$ & $\begin{array}{l}9,4 b \\
0,9\end{array}$ \\
\hline \multirow{3}{*}{$\begin{array}{c}\text { Heure } \\
\text { moyenne } \\
\text { pondérée } \\
\text { d'excrétion } \\
\text { Average hour } \\
\text { of hard faeces } \\
\text { excretion }\end{array}$} & $\begin{array}{l}\text { Jour } \mathbf{I} \\
\text { Day } I .\end{array}$ & . & & $\cdot$ & $\begin{array}{r}2 \mathrm{I}, 9 \\
0,5\end{array}$ & $\begin{array}{l}0,2 \\
1,7\end{array}$ & $\begin{array}{r}-3,3 \\
0,6\end{array}$ \\
\hline & $\begin{array}{l}\text { Jour } 2 \\
\text { Day } 2\end{array}$ & . $\cdot$ & $\cdot$ & $\cdot$ & $\begin{array}{r}22,5 \\
0,5\end{array}$ & $\begin{array}{r}22,9 \\
0,6\end{array}$ & $\begin{array}{r}22,7 \\
0,5\end{array}$ \\
\hline & $\begin{array}{l}\text { Jour } 3 \\
\text { Day } 3\end{array}$ & . & . & 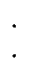 & $\begin{array}{r}22,6 \\
0,7\end{array}$ & $\begin{array}{r}23,8 \\
0,9\end{array}$ & $\begin{array}{l}23,4 \\
0,5\end{array}$ \\
\hline
\end{tabular}



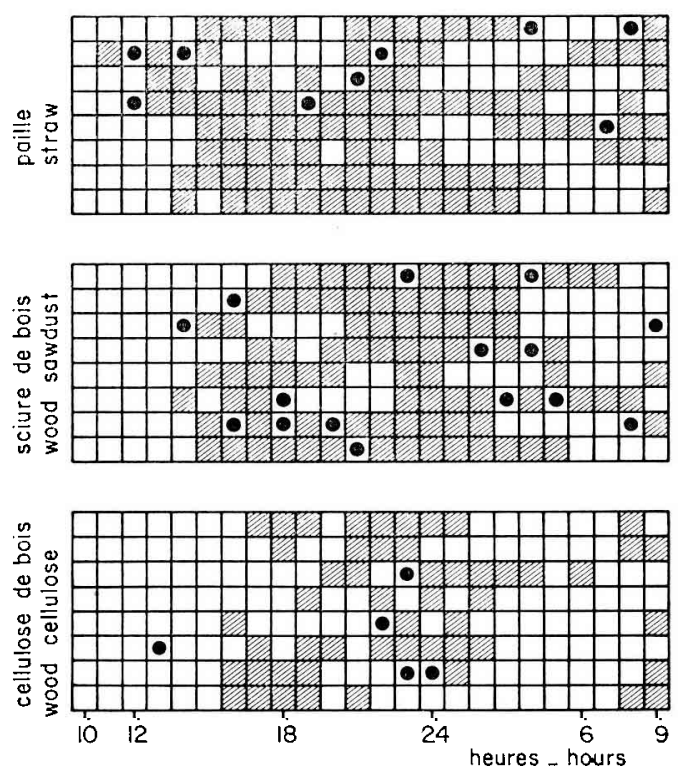

FIG. I. - Diagramme représentatif de l'excrétion fécale de matière sèche (crottes dures) selon la source de cellulose entrant dans la composition de l'aliment.

En noir: collecte positive de plus de $\mathrm{x} g$ de matière sèche.

Points: collecte positive de moins de $\mathrm{I} g$ de matière sèche.

Chaque ligne décrit le second nycthémère expérimental d'un lapin.

Diagram showing faecal excretion of dry matter (hard faeces) according to the kind of cellulose of the diet.

Dark: hours with more than I $\mathrm{g}$ of dry matter excreted.

Points : hours with less than $\mathrm{I} g$ of dry matter excreted.

Each line describes the $2^{\text {nd }}$ experimental day of each rabbit.

\section{TABLEAU 6}

Radioactivité excrétée au cours de chacun des 3 nycthémères expérimentaux et résiduelle dans le tube digestif, en $p$. cent de la radioactivité totale: moyenne et écart-type de la moyenne

Percent of total radioactivity excreted during each of the 3 experimental days

and remaining in the gastro intestinal tract: mean and standard deviation of the mean

\begin{tabular}{|c|c|c|c|}
\hline $\begin{array}{l}\text { Régime } \\
\text { Diet }\end{array}$ & $\begin{array}{l}\text { Paille } \\
\text { Straw }\end{array}$ & $\begin{array}{l}\text { Sciure de bois } \\
\text { Wood sawdust }\end{array}$ & $\begin{array}{l}\text { Cellulose de bois } \\
\text { Wood cellulose }\end{array}$ \\
\hline 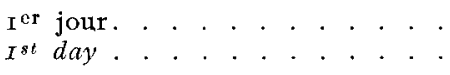 & $\begin{array}{r}78,8 \text { a } \\
2,3\end{array}$ & $\begin{array}{r}68,4 \quad b \\
3,3\end{array}$ & $\begin{array}{c}55,0 \quad c \\
5,5\end{array}$ \\
\hline 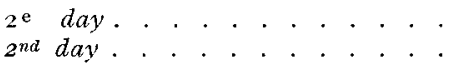 & $\begin{array}{r}\mathbf{1 6}, 0 \quad a \\
\mathrm{I}, 7\end{array}$ & $\begin{array}{r}\mathbf{1} 8,7 a \\
\mathbf{1}, 8\end{array}$ & $\begin{array}{l}\mathrm{I} 9,9 a \\
2,0\end{array}$ \\
\hline 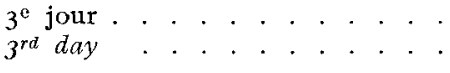 & $\begin{array}{l}4,2 a \\
0,5\end{array}$ & $\begin{array}{ll}8,9 & b \\
1,6 & \end{array}$ & $\begin{array}{r}\mathbf{I}, 5 b \\
\mathbf{I}, 7\end{array}$ \\
\hline $\begin{array}{l}\text { Rad. résiduelle (tube digestif). . } \\
\text { Residual Rad. (G.I. tract). . . . }\end{array}$ & $\begin{array}{l}1,0 \quad a \\
0,2\end{array}$ & $\begin{array}{l}3,9 b \\
\mathbf{I}, 0\end{array}$ & $\begin{array}{r}\text { I } 3,6 c \\
4,3\end{array}$ \\
\hline
\end{tabular}




\section{3. - Elimination $d u{ }^{141} \mathrm{Ce}$}

Les courbes moyennes exprimant sous forme cumulée l'excrétion fécale de la radioactivité (fig. 2) montrent que la rétention du marqueur est beaucoup plus longue chez les lapins ingérant l'aliment cellulose de bois que chez ceux qui reçoivent le régime paille. Un résultat intermédiaire est observé pour le régime sciure de bois.

La courbe représentative de l'excrétion de ${ }^{141} \mathrm{Ce}$ par les lapins recevant 1'aliment cellulose de bois présente un nombre de paliers plus important que les deux autres en raison du morcellement chronologique de l'émission de fèces dures par ces lapins.

Le régime paille conduit à une excrétion massive de la radio-activité au cours des premières 24 heures. L'excrétion la plus faible est le fait du régime cellulose

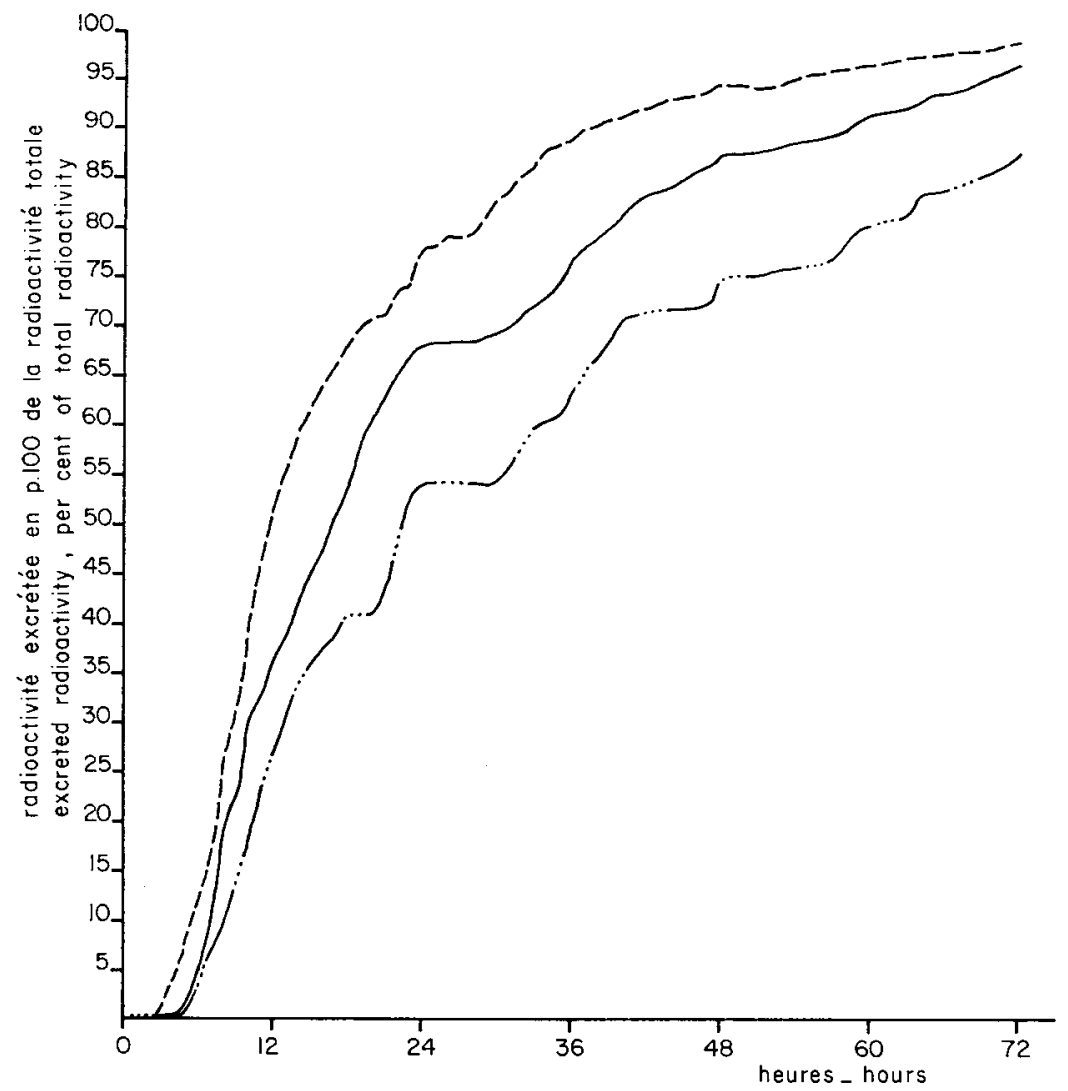

FIG. 2. - Quantités moyennes de radioactivité excrétée cumulée d'heure en heure en $p$. cent de la radioactivité totale.

Cumulative quantities of excreted radioactivity, from hour to hour, as per cent of total radioactivity.

Paille - Straw : -........

Sciure de bois - Wood sawdust:

Cellulose de bois - Wood cellulose: 
de bois pour lequel, à l'inverse, est constatée la plus forte élimination au cours du troisième nycthémère (tab1. 6). Ces particularités sont à relier à celles de l'ingestion d'aliment : la quantité de radioactivité excrétée au cours du I er nycthémère est significativement $(\mathrm{P}<0,05)$ corrélée à la quantité d'aliment ingéré au cours de cette même période dans le cas des régimes paille $(r=0,79)$ et sciure de bois $(r=0,78)$. Par contre cette relation $n^{\prime}$ est pas retrouvée $(r=0,37-\mathrm{N}$.S. $)$ pour le régime cellulose de bois. C'est aussi pour ce dernier régime que la radioactivité résiduelle dans les viscères est la plus élevée.

La répartition de cette radioactivité résiduelle (tabl. 7) est la même pour les compartiments réservoirs (estomac-caecum-côlon) dans les trois groupes. Seul le pourcentage de la radioactivité viscérale retrouvé dans l'intestin grêle est significativement plus élevé avec le régime paille qu'avec les deux autres.

\section{TABIEAU 7}

Répartition de la radioactivité résiduelle dans les 4 principaux compartiments du tractus digestił, exprimée en p. cent de la radioactivité résiduelle totale dans ce derniev. Moyenne et écart-type de la moyenne.

Residual radioactivity in the four main digestive compartments: Per cent of total G.I. tract residual radioactivity. Mean and standard deviation of the mean.

\begin{tabular}{|c|c|c|c|}
\hline $\begin{array}{l}\text { Régime } \\
\text { Diet }\end{array}$ & $\begin{array}{l}\text { Paille } \\
\text { Straw }\end{array}$ & $\begin{array}{l}\text { Sciure de bois } \\
\text { Wood sawdust }\end{array}$ & $\begin{array}{l}\text { Cellulose de bois } \\
\text { Wood cellulose }\end{array}$ \\
\hline 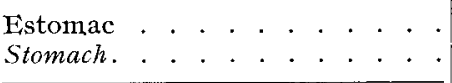 & $\begin{array}{c}2 \mathrm{I}, \mathrm{O} \\
2,3\end{array}$ & $\begin{array}{r}\mathrm{I} 7,7 \\
2,6\end{array}$ & $\begin{array}{c}22,0 \quad a \\
2,3\end{array}$ \\
\hline $\begin{array}{l}\text { Intestin grêle } \\
\text { Small bowel. }\end{array}$ & $\begin{array}{l}7,8 \quad a \\
\mathrm{r}, 6\end{array}$ & $\begin{array}{l}4, \mathrm{I} \quad b \\
\mathrm{O}, 8\end{array}$ & $\begin{array}{l}2,2 b \\
0,4\end{array}$ \\
\hline $\begin{array}{llll}\text { Caecum } & . & . & . \\
\text { Caecum } & . & . & .\end{array}$ & $\begin{array}{l}54,2 \quad a \\
3,7\end{array}$ & $\begin{array}{c}61,7 \quad a \\
2,6\end{array}$ & $\begin{array}{c}58,6 a \\
2,3\end{array}$ \\
\hline 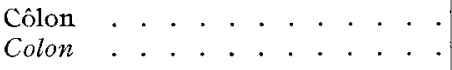 & $\begin{array}{r}\mathrm{I} 6,9 a \\
\mathrm{I}, 2\end{array}$ & $\begin{array}{r}\mathrm{I} 6,5 \\
\mathrm{I}, \mathrm{O}\end{array}$ & $\begin{array}{l}\mathrm{I} 7,0 \quad a \\
\mathrm{r}, 5\end{array}$ \\
\hline
\end{tabular}

\section{Discussion}

Ainsi qu'il était supposé initialement, la nature de la source de cellulose n'est pas indifférente à l'égard du transit digestif chez le lapin en croissance. Dans les conditions de nos régimes isocellulosiques (cellulose de Weende) les trois sources utilisées se classent, par leurs effets, systématiquement dans un même ordre : paille, sciure de bois, cellulose de bois.

On pourrait tenter d'expliquer les différences par la présence des autres constituants membranaires. En effet, les régimes expérimentaux ont des teneurs variables en tanins et en lignine. Mais la plus forte teneur en ces deux éléments est le fait de la sciure de chêne qui conduit toujours aux résultats intermédiaires. De ce fait il semble peu probable que ces constituants soient responsables des effets observés.

La nature exacte de l'influence de la source cellulosique peut être recherchée dans la nature chimique de la cellulose (degré de polymérisation, proportion d'hémi- 
cellulose) ou dans la structure physique des fibres constituant la fraction cellulosique du régime. Sur ce dernier point, un parallèle peut être tenté : nous avons antérieurement constaté que la présence de particules grossières dans l'aliment entraîne une accélération du transit digestif (LAPLACE, LEBAS, I977). On pourrait ici imaginer que le transit soit d'autant plus rapide que la cellulose est apportée sous la forme de fibres plus longues. Malgré l'absence d'une telle mesure pour nos régimes expérimentaux nous pouvons affirmer que la paille et la cellulose de bois purifiée représentent bien deux types diamétralement opposés. Compte tenu de 1'aspect physique de la sciure employée nous sommes en droit de lui supposer une dimension des fibres inférieure à celle de la paille. Un élément favorable à ces hypothèses peut être trouvé dans la proportion d'eau fécale plus importante en présence de fibres plus longues (paille).

On peut aussi envisager que cette influence de la source cellulosique ne soit qu'apparente par le fait d'une mauvaise évaluation quantitative de l'apport de cellulose en raison d'une sur ou sous estimation lors du dosage en fonction de la taille des particules (HELIER, RIVERS et HACKLER, I977). I1 nous paraît néanmoins difficile de relier, dans le cas du régime cellulose de bois, 1'ampleur de la surestimation de la quantité de cellulose apportée et celle du ralentissement du transit constatée par rapport aux autres régimes. L'influence de la structure physique par elle-même reste donc la plus plausible.

Si séduisante que soit l'hypothèse explicative impliquant un effet de la structure physique de la fibre, nous devons rappeler que 1'ordre de classement de nos trois aliments expérimentaux d'après le transit digestif des lapins est identique à leur classement en fonction de leur taux d'amidon. On peut ainsi associer tune teneur élevée en amidon et un transit relativement lent, et inversement. Le mode d'action éventuel de la teneur en amidon resterait alors à préciser.

Une intervention conjointe de ces deux ordres de facteurs (structure, physique de la cellulose - teneur en amidon) est également plausible. On peut noter dans ce sens que la conjonction d'une forte teneur en amidon et de fibres très courtes (aliment cellulose de bois) produit un régime à forte teneur en énergie digestible. A l'inverse, la formulation de 1'aliment paille conduit à une dilution énergétique du régime. Une conséquence directe de ce type d'effet peut du reste être décelée au travers des consommations alimentaires des lapins. Or on sait, au moins dans le cas de l'Homme, (HuNT, STUBBS, I975) que la rapidité de l'évacuation gastrique est ajustée en fonction de la teneur en énergie du régime. Une explication analogue est envisageable ici. En effet, pour les régimes les mieux consommés (paille et sciure de bois) est constatée une corrélation positive entre la vitesse du transit digestif et l'importance de la prise de nourriture. Cette relation n'existe pas pour l'aliment le plus énergétique qui est également consommé en moindre quantité. On peut ajouter à cela le fait que ce même aliment cellulose de bois présente une digestibilité de la matière sèche nettement plus élevée $(75,7 \mathrm{p}$. Ioo) que les 2 autres régimes (paille 66,5 - sciure de bois 65, I p. cent). Ceci conduit une fois de plus (LEBAs et LAPIACE, I977) à constater que 1'aliment le plus digestible, et consommé en moindre quantité, fait aussi l'objet du transit le plus lent.

En conclusion: il s'avère certain que, chez le Lapin, le dosage de la cellulose brute (de Weende) ne permet pas de rendre compte de la présence des constituants membranaires éventuellement responsables des différences de transit digestif. Il apparaît nécessaire dans l'avenir de dissocier cet éventuel effet de celui, également plausible, de la teneur en amidon ou de la concentration énergétique du régime. 


\section{Summary \\ The digestive transit in the rabbit. \\ VIII. Infuence of the source of cellulose}

The influence of three ad libitum fed isocellulosic diets containing either barley straw or oak sawdust, or purified wood cellulose, on the digestive transit, was studied in 24 Californian rabbits of both sexes. After one week of habituation, they received at the age of 8 weeks a dose of $\mathrm{I}_{4} \mathrm{ICe}$, the faecal elimination of which was studied for 72 hours.

According to the results, the fastest rate of passage was recorded with the diet containing straw and the slowest with that including purified wood cellulose. This classification of the three diets based on I 4 I Ce retention was systematically found for all criteria measured. Consequently, the quantitative determination of crude fibre did not take into account the cell wall constituents eventually responsible for these particularities. Various hypotheses are suggested, notably in relation with the starch content and energy concentrations of the diets.

\section{Références bibliographiques}

COLIn M., ARkhurst G., I,EBAS F., i973. Fffets de l'addition de méthionine au régime alimentaire sur les performances de croissance chez le lapin. Ann. Zootech., 22, 485-49I.

Colin M., Mairi Cl., Varssaire Josée, Renaulit L., i976. Etude expérimentale du remplacement dans les aliments pour lapins de la cellulose par des lests minéraux : sable et vermiculite. Rec. Med. Vét., 152, 457-465.

Heir.ER S. N., RIVERS J. M., HACKLER L. R., I977. Dietary fiber: The effect of particle size and $\mathrm{pH}$ on its measurement. $J$. Food Sci., 42, 436-439.

Hun'T J. N., S'TubBs D. F., I975. The volume and energy content of meals as determinants of gastric emptying. J. Physiol., Lond., 245, 209-225.

I,APIACE J. P., LEBAS F., 1975. Le transit digestif chez le lapin. 3) Influence de l'heure et du mode d'administration sur l'excrétion fécale du Cérium I $4^{\mathrm{I}}$ chez le Lapin alimenté ad libitum. Ann. Zootech., 24, 255-265.

LAPLACE J. P., LEBAS F., I977. Le transit digestif chez le Lapin. 7) Influence de la finesse de broyage des constituants d'un aliment granulé. Ann. Zootech., 26, 413-420.

LAPLACE, J. P., LEBas F., Rioperez J., I974. Le transit digestif chez le Lapin. r) Utilisation du Cérium I4 I. Étude méthodologique et descriptive. Ann. Zootech., 23, 555-576.

Leibas F., Laplace J. P., I974. Note : Sur l'excrétion fécale chez le Lapin. Ann. Zootech., 23, $577-58 \mathrm{I}$.

IEBAS F., I,APIACE J. P., I975. Le transit digestif chez le Lapin. 5) T́volution de 1'excrétion fécale en fonction de l'heure de distribution de l'aliment et du niveau de rationnement durant les 5 jours qui suivent l'application de ce dernier. Ann. Zootech., 24, 6r 3-627.

I.EBAS F., LAPIACE J. P., I977. Growth and digestive transit in the rabbit; variations determined by physical form, composition and crude fiber content of the feed. Ann. Biol. anim. Bioch. Biophys. 17, 535-538. 